\title{
DEVELOPMENT AND CHARACTERIZATION OF INDOMETHACIN-LOADED MUCOADHESIVE NANOSTRUCTURED LIPID CARRIERS FOR TOPICAL OCULAR DELIVERY
}

\author{
PATTRAVEE NIAMPREMa,b, S. P. SRINIVAS ${ }^{\mathrm{d}}$, WAREE TIYABOONCHAI ${ }^{\mathrm{a}, \mathrm{b}, \mathrm{c}^{*}}$
}

aFaculty of Pharmaceutical Sciences, Naresuan University, Phitsanulok, Thailand, bThe Center of Excellence for Innovation in Chemistry (PERCH-CIC), Commission on High Education, Ministry of Education, Thailand, cThe Center of Excellence in Medical Biotechnology, Naresuan University, Phitsanulok, Thailand, dSchool of Optometry, Indiana University, Bloomington, IN, United States

Email: wareet@nu.ac.th

Received: 12 Jan 2018, Revised and Accepted: 13 Feb 2018

\section{ABSTRACT}

Objective: To develop and characterize indomethacin loaded-nanostructured lipid carriers (IND-NLCs) for topical ophthalmic delivery with different particle sizes and polymer coating to improve the mucoadhesive property on the ocular surface.

Methods: Nanostructured lipid carriers (NLCs) with different solid lipids and surfactants were prepared by the high-pressure homogenization technique. The optimized IND-NLCs was coated with polyethylene glycol 400 (PEG). The physicochemical properties and entrapment efficacy (EE) were examined. In vitro release studies were investigated using the shake-flask method. Ex vivo mucoadhesive studies were assessed by the washoff test. In addition, the cytotoxicity was assessed by the short time exposure test.

Results: IND-NLCs of $\sim 300$ and $\sim 40 \mathrm{~nm}$ in diameter were successfully produced with a zeta potential of $-30 \mathrm{mV}$ and EE of $60-70 \%$. IND-NLCs prepared with Tween 80 as surfactant could be sterilized by autoclaving. The PEG coating of IND-NLCs did not affect either the particle size or EE. In vitro release showed a prolonged release for $360 \mathrm{~min}$ with a burst release of 50-60\% occurring within 5 min. The smaller-sized IND-NLCs showed slightly faster release rates and better mucoadhesion to cornea compared to the larger IND-NLCs. PEG-coated IND-NLCs showed the highest mucoadhesion. In addition, IND-NLCs showed less cytotoxicity compared to IND alone.

Conclusion: The small and PEG-coated NLCs represents a potentially useful carrier for safe delivery of indomethacin to the ocular surface with increased residence time.

Keywords: Indomethacin, Topical ocular delivery, Nanostructured lipid carriers, Polyethylene glycol, Mucoadhesion

(C) 2018 The Authors. Published by Innovare Academic Sciences Pvt Ltd. This is an open access article under the CC BY license (http://creativecommons.org/licenses/by/4.0/) DOI: http://dx.doi.org/10.22159/ijap.2018v10i2.24738

\section{INTRODUCTION}

Indomethacin (IND), a common non-steroidal anti-inflammatory drug (NSAID) in ocular therapeutics, is administered topically on the ocular surface for clinical management of conjunctivitis, anterior uveitis, and post-operative inflammation following cataract surgery [1]. Although it is stable at lower $\mathrm{pH}$, IND possesses a low solubility of $3-5 \mathrm{mg} / 100 \mathrm{ml}$ at $\mathrm{pH} 5.6$ [2]. IND can be rendered soluble in aqueous buffers at $\mathrm{pH}$ 7.5-8.0 but with a risk of its hydrolysis into 5methoxy-2-methyl indolyl-3-acetic acid and 4-chlorobenzene acid [3]. Therefore, IND is formulated in the form of gels and suspensions to improve the solubility and stability $[2,3]$. A polyethylene glycol (PEG)-based formulation of $0.1 \%$ indomethacin solution (Indocollyre ${ }^{\circledR}$ ) is commercially available but it is known to exhibit poor bioavailability and cause irritation, superficial punctuate keratitis, and local pain [4]. Accordingly, nano-carriers drug delivery systems have been proposed as potential alternatives [5-8].

Nano-carrier systems, in general, are able to encapsulate drug and thereby protect them against degradation. Also, nanoparticles can be mucoadhesive, and accordingly show increased their retention time on the ocular surface. These characteristics are suitable for enhanced stability and topical bioavailability of IND [9]. Among the nano-carrier systems, nanostructured lipid carriers (NLCs) possess unique characteristics that are promising for ophthalmic drug delivery. They have been developed to combine the advantages of other colloidal carriers but without their disadvantages [10]. For example, NLCs can be prepared using physiological lipids similar to nanoemulsions and liposomes, thus, providing less significant toxicity and acidic related inflammation problems compared to polymeric nanoparticles such as PLGA nanoparticles [11, 12]. Moreover, NLCs show better physical stability than nanoemulsions and liposomes by remaining in a solid state at room and body temperature. NLCs are composed of the imperfect crystalline structure of lipid matrix which formed by a mixture of solid and liquid lipid, and stabilized by surfactants. Therefore, the drug payload is increased, and expulsion of the drug during storage is avoided as compared to solid lipid nanoparticles (SLNs) [13].

A major challenge of topical ocular drug delivery is the poor bioavailability of the drug at the ocular surface and the anterior chamber [12]. Employing nano-carrier systems, the topical bioavailability can be improved by enhancing their residence time and corneal epithelial uptake [12]. Both parameters are strongly affected by the particle size and surface modification [11]. Although, Balguri et al. have recently reported increased bioavailability of IND to ocular tissues with IND-loaded NLCs [7], the effect of particle size and surface modifications have not been delineated.

Therefore, the aim of this study was to develop IND-loaded NLCs (IND-NLCs) with different types of solid lipids and surfactants which improve the mucoadhesive property on the corneal surface. Surfacemodification of IND-NLCs with polyethylene glycol 400 (PEG) was also undertaken. Physicochemical properties of the developed INDNLCs were evaluated in terms of particle size, zeta potential, drug entrapment efficacy, and in vitro release study. The effect of the autoclaving method on the physicochemical stability of IND-NLCs was investigated. The retention of IND-NLCs on porcine cornea mucosa was determined using ex vivo mucoadhesive studies. In addition, in vitro cytotoxicity of the optimized IND-NLCs was investigated by the short time exposure test (STE) in primary porcine corneal epithelial (PCE) cells.

\section{MATERIALS AND METHODS}

\section{Materials}

Gelucire 44/14 (lauryl macrogol-32 glyceride) and Compritol 888 ATO (Compritol, glyceryl behenate) were kindly gifted by Gattefossé (Cedex, France). Lexol GT865 (medium chain triglyceride), cetyl palmitate and squalene were purchased from Namsiang trading 
(Bangkok, Thailand). Tween 80 (Polysorbate 80) was acquired from AjexFinechem (Sydney, Australia). Emulmetik 900 was purchased from Lucas Meyer (Ludwigshafen, Germany). Indomethacin (Lot BCBK0293) was acquired from Sigma-Aldrich (China). AmiconUltra $10 \mathrm{~K}$ centrifugal filter was kindly gifted from Merck Millipore (Massachusetts, USA). Methanol and Acetonitrile used as HPLC solvent were purchased in HPLC grade quality from Lab Scan.

Keratinocyte serum-free medium (K-SFM), bovine pituitary extract, recombinant human epidermal growth factor (EGF), and Gibco antibiotic-antimycotic (100x) were purchased from Invitrogen (California, USA). Hydrocortisone solution, human insulin solution, bovine serum albumin (BSA), bovine collagen type I, and human fibronectin were obtained from Sigma-Aldrich (Steinheim, Germany).

\section{Preparation of blank-NLCs and IND-NLCs}

IND-NLCs were prepared by a high-pressure homogenization technique. Briefly, the lipid phase contained $50 \mathrm{mg}$ of IND, $2 \%$ $(\mathrm{w} / \mathrm{w})$ of squalene, $2 \%(\mathrm{w} / \mathrm{w})$ of Emulmetik $900,2 \%(\mathrm{w} / \mathrm{w})$ of Lexol GT865, and $3 \%(\mathrm{w} / \mathrm{w})$ of solid lipid was heated at $80^{\circ} \mathrm{C}$. Meanwhile, the aqueous phase consisting of $7 \%(\mathrm{w} / \mathrm{w})$ of surfactant dissolved in distilled water was heated at $80^{\circ} \mathrm{C}$ and slowly added to the oil phase. The obtained a primary emulsion was subjected to a high-speed homogenizer at $5000 \mathrm{rpm}$ (T18, IKA, Staufen, Germany) for $1 \mathrm{~min}$ before subjected to a high-pressure homogenizer (M-110P, Microfluidics, Massachusetts, USA) applying 5 cycles at 1500 bar.

The resulting hot o/w microemulsion was cooled at $25{ }^{\circ} \mathrm{C}$, resolidification the lipid and forming the NLCs. Finally, the obtained NLCs was washed twice with a normal saline solution (NSS) using an ultrafiltration system (Amicon 8400, Massachusetts, USA) fitted with a molecular weight cut off 100 $\mathrm{kDa}$ membrane to remove the excess components and unencapsulated IND. Blank-NLCs were prepared with the same method as described above without adding IND. NLCs were prepared by varying the type of solid lipid and surfactant namely; NLC1 (Compritol888 ATO and Tween 80); NLC2 (cetyl palmitate and Tween 80); NLC3 (Compritol888 ATO and Gelucire 44/4); and NLC4 (cetyl palmitate and Gelucire 44/4). The selection of these variables was based on preliminary experiments.

To prepare the mucoadhesive NLCs, NLC2 and IND-NLC2 were incubated with $0.1 \%(\mathrm{w} / \mathrm{v})$ PEG 400 at a ratio of $1: 1$ and stirred at $500 \mathrm{rpm}$ for $30 \mathrm{~min}$ (C-MAG HS7, Guangzhou, China). The obtained polymer coated NLC2 (NLC2-PEG) and polymer-coated IND-NLC2 (IND-NLC2-PEG) were washed as described above. All samples were prepared in triplicate.

\section{Sterilization by autoclaving}

Blank-NLCs and IND-NLCs were placed in a glass vial and sealed with rubber stoppers and aluminium caps. Then, the samples were sterilized by steam sterilization at $121^{\circ} \mathrm{C}$ for $15 \mathrm{~min}$ at 2 bar. After the autoclaving process, the sterilized formulations were characterized the particle size, zeta potential, and drug remaining (\%).

\section{Drug incorporation efficiency}

The content of IND incorporated into NLCs was determined by extraction method. Briefly, $100 \mu \mathrm{l}$ of IND-NLCs was mixed with $900 \mu \mathrm{l}$ of a mixture of $1 \mathrm{M} \mathrm{HCl}$ and methanol $(1: 90, \mathrm{v} / \mathrm{v})$ and sonicated at $40 \%$ amplitude for 30 seconds by the ultrasonic probe (VCX130, Connecticut, USA). The sample was then centrifuged at 31,514 $\times$ g for $10 \mathrm{~min}$; then the supernatant was collected to determine the amount of IND using a modified high-performance liquid chromatography (HPLC) assay of Nováková L et al. [14]. The HPLC system (LC10AT, Shimadzu, Kyoto Japan) composed of an autosampler model SIL-10ADVP, a pump system model LC20-AT, and a UV/VIS detector model SPD-20A. Twenty $\mu \mathrm{l}$ of the

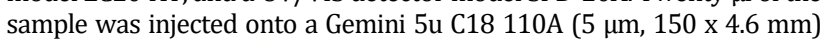
which was kept at $25^{\circ} \mathrm{C}$. The mobile phase consisted of acetonitrile and $0.2 \%(\mathrm{v} / \mathrm{v})$ orthophosphoric acid at the volume ratio of $65: 35(\mathrm{v} / \mathrm{v})$ was delivered at a flow rate of $1.2 \mathrm{ml} / \mathrm{min}$. The UV detection was set at 270 $\mathrm{nm}$. IND was quantified from its peak area using calibration curve of IND established from the range of 1 to $50 \mu \mathrm{g} / \mathrm{ml}$. The percentage of drug incorporation efficiency was calculated as [(Amount of extracted IND) $\times$ $100] /$ (Initial amount of IND).

\section{Physico-chemical characterization of blank-NLCs and IND-NLCs}

The mean particle size and polydispersity index (PI) were determined by dynamic light scattering (DLS) with a ZetaPALS ${ }^{\circledR}$ analyzer (Brookhaven 90Plus, New York, USA). This instrument was equipped with a $35 \mathrm{~mW}$ HeNe laser diode operating at $632.8 \mathrm{~nm}$ and a BI-200SM Goniometer connected to a BI-9010AT digital correlator. Samples were dispersed in DI water and run for 10 measurement cycles. The mean particle size and PI values were obtained by the auto measuring mode at a fixed angle of $90^{\circ}$. All samples were performed in triplicate.

The zeta potential determined by measuring the particle electrophoretic mobility using the ZetaPAL ${ }^{\circledR}$ analyzer. The measurement was then carried out at $25^{\circ} \mathrm{C}$ and angle of $14.8^{\circ}$ to the incident light. The zeta potential was calculated based on the Smoluchowski equation. The measurement was performed for 5 cycles.

The transmission electron microscope (TEM, Tecnai 12, Philips, USA) was used to examine the morphology of NLCs and IND-NLCs by negative staining method. Twenty $\mu \mathrm{l}$ of sample was deposited on a carbon-coated 300 mesh copper grid. Then, $10 \mu \mathrm{l}$ of $0.5 \%(\mathrm{w} / \mathrm{v})$ uranyl acetate in ethanol was dropped onto the grid. The excessive solvent was removed by Whatman no.1 filter paper and allowed to air-dry at room temperature. The dried sample was kept in a desiccator for further observation by TEM.

\section{In vitro release study}

The shake-flask method was employed to evaluate the dissolution profile of IND-NLCs formulations [15]. IND is a poorly water-soluble drug. Therefore, NSS (pH 5.5) contained $0.6 \%(\mathrm{v} / \mathrm{v}$ ) Tween 80 was used as a dissolution medium to provide sink condition [16]. One ml of IND-NLCs (containing IND $0.4 \mathrm{mg}$ ) was mixed into $10 \mathrm{ml}$ of dissolution medium and stirred at $200 \mathrm{rpm}$ at $34 \pm 0.5^{\circ} \mathrm{C}$. An aliquot $(500 \mu \mathrm{l})$ of the sample was taken at pre-determined time intervals of $5,30,60,120,180,240$, and $360 \mathrm{~min}$. The fresh medium was replaced immediately after sampling to maintain a constant volume. Samples were then centrifuged using $10 \mathrm{~K}$ Amicon centrifugal filter at $17,508 \times \mathrm{g}$ for $10 \mathrm{~min}$. The filtrate was collected and mixed with mobile phase before performed on HPLC system as described above.

\section{Ex vivo mucoadhesive study}

The retention of IND-NLCs on the corneal surface was determined using an experimental setup previously described by Chaiyasan $e t$ al. [17]. Porcine eyes were obtained from the local slaughterhouse. The eyes were kept on the ice and kept moist with $1 \%(\mathrm{v} / \mathrm{v})$ antibiotic solution until use $(<8 \mathrm{~h}$ after death). The corneal tissue was cut out with a trephine ( $6 \mathrm{~mm}$ diameter) and mounted on a glass slide. INDNLCs $(10 \mu \mathrm{l})$ was instilled on the cornea surface. Then, the tissue was exposed to a continuous stream of pH $5.5 \mathrm{NSS}, 34{ }^{\circ} \mathrm{C}$, at a flow rate of $0.3 \mathrm{ml} / \mathrm{min}$ for $5,15,30$, and $60 \mathrm{~min}$ to induce shear stress mimicking blink action. Finally, the cornea tissue was collected and extracted to determine the IND-NLCs adhered to the tissue using HPLC method. The percentage of IND retained on the ocular surface calculated as [(Amount of extracted IND from cornea tissue) $\times$ $100] /$ (Initial amount of IND-NLCs).

\section{Cytotoxicity of IND-NLCs in primary porcine corneal epithelial (PCE) cells}

Primary porcine corneal epithelial (PCE) cells from primary tissue explant technique were cultured as before $[18,19]$. Briefly, the cornea was excised from the freshly isolated porcine eye, then sterilized with $1 \%(\mathrm{v} / \mathrm{v})$ povidone-iodine for $5 \mathrm{~min}$ and rinsed thrice with PBS containing $1 \%(\mathrm{v} / \mathrm{v})$ antibiotic-antimycotic. The explant was then placed epithelial side down onto 6-well tissue pre-coated culture plate with coating solution contained BSA, bovine collagen I, and human fibronectin. The explant was further cultured in K-SFM with supplements at $37^{\circ} \mathrm{C}$, in a humidified atmosphere containing $5 \% \mathrm{CO}_{2}$. The cornea was removed after $5 \mathrm{~d}$, and the outgrowing cells were maintained in culture medium for 2-3 w.

For several years, the method of choice to determine eye irritation potential was the Draize rabbit eye test. However, ethical considerations and the limited value of animal models including lack of reproducibility and overestimation of human response led to the development of alternative in vitro tests [20]. The short time exposure (STE) in vitro test is recommended for assessing eye irritation potential. The STE method is 
straightforward to use and known to provide rapid results with the excellent predictive ability [21].

The cytotoxicity was assessed by the STE test using MTT assay as described by Kojima et al. but with some modifications [20]. The PCE cells were seeded on 96-well plate at $2 \times 10^{4}$ cells/well until reaching the semi-confluence (2-3 d). Then, the cells were exposed to $200 \mu \mathrm{l}$ of IND-NLCs dispersed in PBS containing 5 and $0.05 \%(\mathrm{w} / \mathrm{v})$ indomethacin for $5 \mathrm{~min}$ at room temperature. In addition, 5 and $0.05 \%(\mathrm{w} / \mathrm{v})$ IND solution, PBS, and $0.01 \%(\mathrm{w} / \mathrm{v})$ sodium lauryl sulfate (SLS) were used as sample test, vehicle, and positive control, respectively. After exposure, the cells were washed with PBS twice, and $200 \mu \mathrm{l}$ of methyl thiazol diphenyl-tetrazolium bromide (MTT) solution $(0.5 \mathrm{mg} / \mathrm{ml})$ in culture medium was added. After incubated for $2 \mathrm{~h}$, MTT formazan was extracted with $200 \mu \mathrm{l}$ of a mixture of 0.04 $\mathrm{N} \mathrm{HCl}$ in absolute isopropanol and DMSO $(1: 1, \mathrm{v} / \mathrm{v})$ for $30 \mathrm{~min}$. The absorbance was measured at $570 \mathrm{~nm}$ with a microplate reader (BioStack Ready, Vermont, USA). The relative cell viability was calculated comparing to the vehicle, then, category and rank classification was determined as described by Takahashi et al. [22].

\section{RESULTS}

\section{Physicochemical characteristics of blank-NLCs}

Table 1 shows the effect of different solid lipids, surfactants, and PEG coating on the physical characteristics of NLCs. NLC1 and NLC3, prepared with Compritol 888 ATO as solid lipid, showed a larger particle size of $307 \pm 29$ and $144 \pm 8 \mathrm{~nm}$, respectively. While preparing with cetyl palmitate, NLC2 and NLC4, reduced the particle size to $39 \pm 4$ and $34 \pm 1 \mathrm{~nm}$, respectively.

Moreover, NLC1 and NLC2, prepared with Tween 80 as a surfactant, demonstrated a larger particle size compared to those with Gelucire 44/14, NLC3 and NLC4. The PI value of all formulations was less than 0.3 indicating a narrow size distribution. The hydrophilic coating with PEG, NLC2-PEG, affected neither the particle size nor zeta potential $(\mathrm{p}>0.05)$.

Table 1: The components and physicochemical properties of NLCs

\begin{tabular}{llll}
\hline Formulations & Type of solid lipid: surfactant & $\begin{array}{l}\text { Mean size (nm) } \pm \text { SD } \\
\text { before autoclave } \\
\text { (after autoclave) }\end{array}$ & $\begin{array}{l}\text { Polydispersity index } \pm \text { SD } \\
\text { before autoclave } \\
\text { (after autoclave) }\end{array}$ \\
\hline NLC1 & Compritol: Tween 80 & $307 \pm 29(261 \pm 10)$ & $0.24 \pm 0.02(0.31 \pm 0.03)$ \\
NLC2 & cetyl palmitate: Tween 80 & $39 \pm 4(40 \pm 3)$ & $0.34 \pm 0.02(0.21 \pm 0.08)$ \\
NLC2-PEG & cetyl palmitate: Tween 80 & $42 \pm 2(37 \pm 3)$ & $0.16 \pm 0.05(0.19 \pm 0.03)$ \\
NLC3 & Compritol: Gelucire 44/14 & $144 \pm 8(\mathrm{PS})$ & $-27 \pm 1(-26 \pm 2)$ \\
NLC4 & cetyl palmitate: Gelucire $44 / 14$ & $34 \pm 1(\mathrm{PS})$ & $-30 \pm 3(-24 \pm 3)$ \\
\hline
\end{tabular}

SD: standard deviation for $n=3$; PS: phase separation

The effects of sterilization by autoclaving on the physical characteristics of the blank-NLCs formulations were presented in table 1 . The surfactant used in the formulations showed a critical effect on physical stability. NLC3 and NLC4, prepared by Gelucire $44 / 14$ could not autoclave as evidenced by the appearance of oil droplets, phase separation, and particle aggregation. However, NLC1, NLC2, and NLC2-PEG, prepared with Tween 80, provided stable NLCs. No significant difference in all the parameters was observed before and after autoclaving ( $p>0.05)$.

\section{Physicochemical characterization of IND-NLCs}

Table 2 showed the effect of different solid lipids, surfactants, and PEG coating on the physical characteristics of IND-NLCs. Compared to blank NLCs, IND-NLCs were slightly larger $(\mathrm{p}<0.05)$. However, IND loading showed no significant influence on the zeta potential and PI $(p>0.05)$. The entrapment efficiency (EE) of IND-NLC1, INDNLC2, and IND-NLC2-PEG was 74.11 $\pm 2.81,65.09 \pm 3.16$, and $62.75 \pm 4.10$, respectively.

Table 2 also showed lack of any significant effect of sterilization on the physicochemical characteristics of all IND-NLCs formulations.

The morphology examined by TEM was shown in fig. 1, both uncoated IND-NLC2, and PEG-coated IND-NLC2 exhibited spherical shape. The light grey border at the periphery (fig. 1B) was attributed to the presence of PEG coating around the particle surface.

Table 2: Physicochemical characterizations of the optimized IND-NLCs

\begin{tabular}{lllll}
\hline Formulations & $\begin{array}{l}\text { Mean size }(\mathbf{n m}) \pm \text { SD } \\
\text { before autoclave } \\
\text { (after autoclave) }\end{array}$ & $\begin{array}{l}\text { Polydispersity index } \mathbf{S D} \\
\text { before autoclave } \\
\text { (after autoclave) }\end{array}$ & $\begin{array}{l}\text { Zeta potential (mV) } \pm \text { SD } \\
\text { before autoclave } \\
\text { (after autoclave) }\end{array}$ & $\begin{array}{l}\text { Entrapment efficacy } \\
\text { before autoclave } \\
\text { (after autoclave) }\end{array}$ \\
\hline IND-NLC1 & $333 \pm 19(261 \pm 10)$ & $0.27 \pm 0.05(0.31 \pm 0.03)$ & $-25 \pm 5(-26 \pm 2)$ & $74.11 \pm 2.81(73.91 \pm 0.37)$ \\
IND-NLC2 & $46 \pm 5(40 \pm 3)$ & $0.34 \pm 0.06(0.21 \pm 0.08)$ & $-26 \pm 4(-24 \pm 3)$ & $65.09 \pm 3.16(61.10 \pm 2.84)$ \\
IND-NLC2-PEG & $43 \pm 2(40 \pm 3)$ & $0.12 \pm 0.02(0.21 \pm 0.08)$ & $-28 \pm 1(-24 \pm 3)$ & $62.75 \pm 4.10(60.35 \pm 1.49)$ \\
\hline
\end{tabular}

SD: standard deviation for $\mathrm{n}=3$
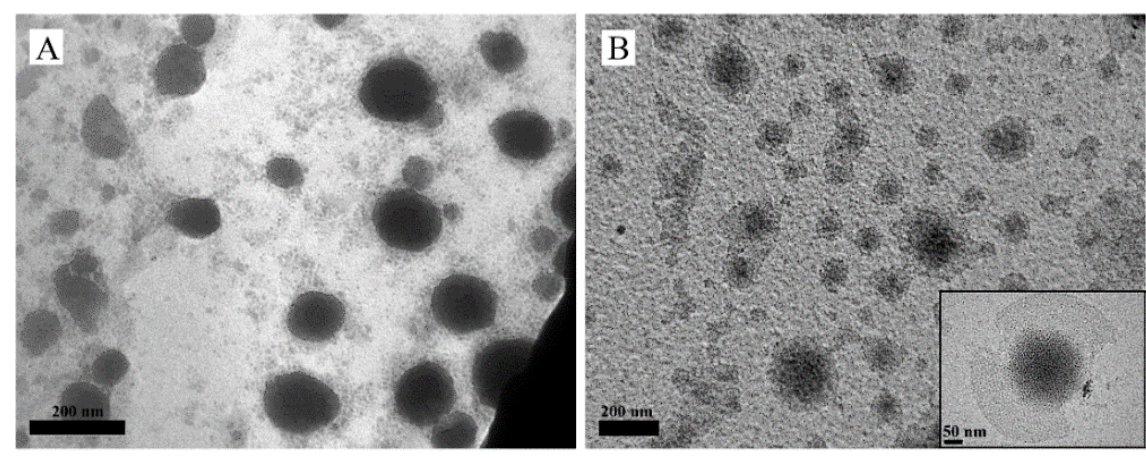

Fig. 1: TEM micrographs of (A) IND-NLC2 and (B) IND-NLC2-PEG 


\section{In vitro release study}

IND has a poor solubility of $0.83 \pm 0.04 \mu \mathrm{g} / \mathrm{ml}$ in NSS at ambient temperature. Therefore, $0.6 \%(\mathrm{w} / \mathrm{v})$ Tween 80 was added to the NSS to provide a sink condition by increasing the solubility of IND to $206.67 \pm 7.08 \mu \mathrm{g} / \mathrm{ml}$. As shown in fig. 2, all formulations showed a biphasic release profile with a burst release during 5 min followed by a prolonged release up to $6 \mathrm{~h}$ following Higuchi's model (Supplemental table 1). At the first $5 \mathrm{~min}$, the cumulative release of IND from the INDNLC1 and IND-NLC2 was $48.24 \pm 3.15$ and $56.14 \pm 9.86 \%$, respectively. Then, they increased to $93.89 \pm 4.76$ and $98.25 \pm 4.20 \%$, respectively, after $6 \mathrm{~h}$. Moreover, in the case of IND-NLC2-PEG, the cumulative released of IND at 5 and 360 min was $47.64 \pm 0.92$ and $80.04 \pm 4.72 \%$, respectively. The results showed that the smaller particle size showed faster drug release rate than larger ones. Moreover, IND-NLC2-PEG showed slower drug release rate than uncoated ones (IND-NLC2).

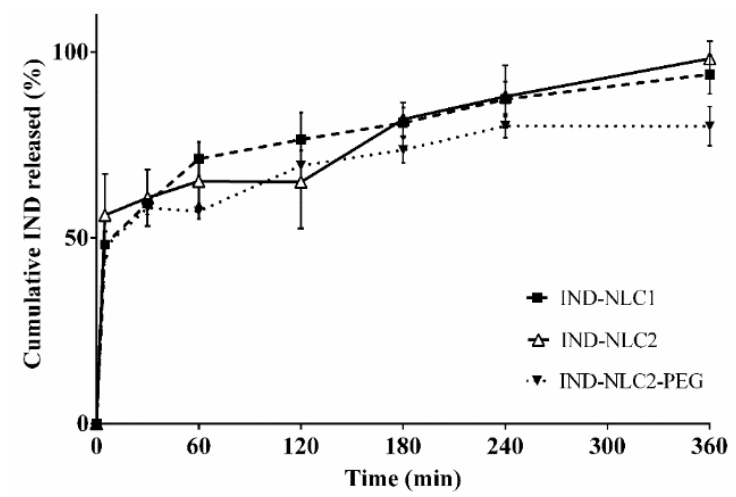

Fig. 2: In vitro release profiles of IND from IND-NLC1, IND-NLC2, and IND-NLC2-PEG in NSS with $0.6 \%(w / v)$ Tween 80 at $34{ }^{\circ} \mathrm{C}$. Error bars represent standard deviation for $n=3$

\section{Ex vivo mucoadhesion test}

The percentage of IND remaining on the porcine cornea tissue after installation was depicted in fig. 3. After 5 min of fluid flow, cornea tissue instilled with IND solutions showed $\sim 28 \%$ remaining while those instilled with IND-NLC1, IND-NLC2, and IND-NLC2-PEG showed $\sim 59 \%, \sim 70 \%$, and $\sim 73 \%$ remaining, respectively. Moreover, nearly $100 \%$ loss occurred after $60 \mathrm{~min}$ of fluid flow when instilled with IND solutions, IND-NLC1, and IND-NLC2. On the other hand, IND-NLC2-PEG showed $\sim 8 \%$ remaining after 60 min of continuous fluid flow. These results indicated that small NLCs (INDNLC2) showed higher mucoadhesive property than larger NLCs (IND-NLC1), especially, NLCs coating with PEG provided the highest mucoadhesiveness on ocular surface.

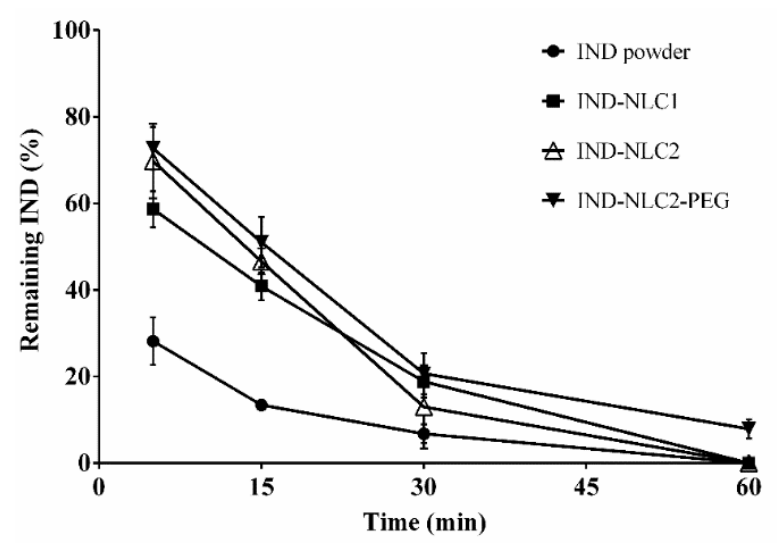

Fig. 3: The percentage of IND remaining on porcine cornea tissue after a steady flow of NSS for $60 \mathrm{~min}$. Error bars represent standard deviation for $n=3$

\section{Cytotoxicity of IND-NLCs}

As shown in table 3, exposure of PCE cells to IND-NLC2 and INDNLC2-PEG contained 0.5 and 5\% IND, showed cell viability of $>70 \%$. Based on Takahashi et al., they could be classified as non-irritants [22]. On the other hand, 5\% of IND-solutions and $0.01 \%$ SLS (positive control) exhibited cell viability (\%) of $56.16 \pm 4.23$ and $59.69 \pm 3.96$, respectively, confirming their irritation potential [22].

Table 3: Summary of short time exposure (STE) tests performed by MTT assay

\begin{tabular}{|c|c|c|c|}
\hline Sample & IND (\% w/v) & Cell viability (\%) (mean \pm SD) & STE classification $^{a}$ \\
\hline \multirow[t]{2}{*}{ IND-NLC2 } & 5 & $72.47 \pm 1.51$ & non-irritant \\
\hline & 0.05 & $92.76 \pm 2.54$ & non-irritant \\
\hline \multirow[t]{2}{*}{ IND-NLC2-PEG } & 5 & $73.63 \pm 1.05$ & non-irritant \\
\hline & 0.05 & $91.24 \pm 4.81$ & non-irritant \\
\hline \multirow[t]{2}{*}{ IND-solution } & 5 & $56.16 \pm 4.23$ & irritant \\
\hline & 0.05 & $72.56 \pm 2.47$ & non-irritant \\
\hline SLS & 0.01 & $59.69 \pm 3.96$ & irritant \\
\hline
\end{tabular}

aEye irritation potential classification STE; Cell viability $>70 \%$ is classified as non-irritant [21], SD: standard deviation for $n=3$

\section{DISCUSSION}

Topical NSAIDs frequently produce side effects and adverse reactions ranging from burning sensation, stinging, and to minor signs of ocular irritation [23]. Stroobant et al., reported several formulations of topical NSAIDs eye drops to impact the rabbit corneal epithelial adversely [24]. Hence, safe and effective formulations of topical NSAIDs remain an unmet need in ophthalmic therapeutics [25]. In this study, NLCs was employed as a drug carrier with the goal not only to overcome the adverse effects of topical NSAIDs but also to provide enhanced bioavailability, which could be improved by enhanced residence time on the cornea surface.

IND-NLCs were successfully prepared by a high-pressure homogenization technique. This technique has been used extensively because of its advantages including reliability, ease of scale-up, and cost-effectiveness [26]. The particles produced were found to be suitable for ophthalmic applications with size ranging $\sim 40-300 \mathrm{~nm}$. In addition, all INC-NLCs formulations exhibited a high zeta potential of $-30 \mathrm{mV}$, which would provide a long-term physical stability. The negative charge could be attributed to the presence of medium chain triglyceride carboxylic group on the particle surface [27].

In agreement with previous reports, the number of the fatty acid side chain on solid lipid and surfactant had a significant effect on the particle size of NLCs $[28,29]$. In case of solid lipid type, NLCs prepared with Compritol 888 ATO (fatty acid side chains, $\mathrm{C}_{22}$ ) resulted in larger particle size than NLCs prepared with cetyl palmitate (fatty acid side chains, $\mathrm{C}_{16}$ ). Similarly, NLCs stabilized with Tween 80 (fatty acid side chains, $\mathrm{C}_{18}$ ), showed larger size than NLCs stabilized with Gelucire 44/14 (fatty acid side chains, $C_{12}$ ). These could be attributed to the solid lipid, and surfactant with long fatty acid side chain commonly forms larger particle size. 
The type of liquid lipid, solid lipid and surfactant all play an important role in incorporating a drug into NLCs [30]. In general, the incorporation of a liquid lipid consisting of a medium chain triglyceride along with a solid lipid consisting of a long chain triglyceride is known to increase the loading capacity and also enable controlled release [31]. Therefore, Lexol GT865 (medium chain triglyceride, $\mathrm{C}_{8}$ and $\mathrm{C}_{10}$ ) was chosen as a liquid lipid in all formulations [32]. As expected, IND-NLC1 prepared with Compritol 888 ATO (fatty acid side chains, $\mathrm{C}_{22}$ ) showed higher EE compared to IND-NLC2 prepared with cetyl palmitate (fatty acid side chains, $\mathrm{C}_{16}$ ). This could be explained by increasing the space created between the solid fatty acid chain and the medium chain triglyceride, allowing more drug to be accommodated [33,34]. In addition, the complete IND incorporation was confirmed by polarized light microscopy (data not shown).

Sterilization of ophthalmic formulation is critical for topical application as microbial contamination should be prevented [35, 36]. One of the strongest advantages of these NLCs is possible to sterile by an autoclaving method which is a commonly used and reliable technique. Taken together, the components for the preparation of NLCs must withstand the conditions of sterilization. As shown in table 1, the physicochemical properties of NLCs were not affected by the type of solid/liquid lipids and polymer after autoclaving. However, the type of surfactant appears to have a profound influence. We found that NLCs prepared with Tween 80 withstood the sterilization by autoclaving in agreement with previous reports $[37,36]$. The phenomena could be explained by the difference in the fatty acid side chain of the surfactants. During autoclaving, the lipid matrix melts and then recrystallizes again during cooling at room temperature. As Tween 80 has a side chain of 18 carbon atoms, its long chain could have more chance to penetrate to the solid lipid phase, consequently lead to more compact and stronger particles. Gelucire $44 / 14$, on the other hand, is composed of 12-carbon short side chain and hence it would be harder to stabilize the particles leading to phase separation.

Depending on the production process and especially different lipid blended, the different type of NLCs are obtained [26]. Due to the ratio of solid lipid/oil, 5/4, used in developed NLCs formulations, we assumed that the multiple types of NLCs would be achieved [31]. According to Müller et al., high levels of the oil can exceed their solubility in the solid lipid leading to precipitation as tiny oil nano compartments within the lipid matrix during the cooling process [26]. These oil nano compartments can contain a higher amount of IND. However, the release of the drug would still be controlled by the surrounding solid lipid barrier. These observations help explain in vitro release data as discussed below.

As shown in fig. 2, we found biphasic IND release, with the burst phase contributing up to $50 \%$. This burst release arguably would be beneficial since therapeutic drug levels can be reached after the administration [39]. The burst release of IND could be attributed to the re-distribution of IND during preparation process [26]. During hot homogenization, heating leads to an increased IND solubility in the water phase, thus, some drug partition from the melt lipid droplet to the water phase. However, during cooling, the solubility of the drug in water phase decreases leading to a re-partitioning of the drugs into the lipid phase. At the same time, the lipid phase starts to solidify and thus, the drugs are not accessible for the re-partitioning. Therefore, some of IND are accumulated at the matrix surface leading to the burst release characteristics.

However, after the burst release, the release profile could be the best fit to the Higuchi square root model. This indicates that IND released from the NLCs in the second phase occurs by a diffusion-controlled mechanism from oil nano-compartment to the matrix for subsequent release to the medium. Also, the release of IND-NLC2 coated with PEG, an uncharged hydrophilic polymer, is slightly reduced possibly due to the presence of the polymer in the outer regions of the particles.

IND-NLCs showed grater adhesion on the porcine cornea tissue compared to IND solutions. It was found that the small IND-NLC2 $(\sim 40 \mathrm{~nm})$ showed higher retention than large IND-NLC1 $(\sim 300 \mathrm{~nm})$. Li et al., had suggested that NLCs with a particle size of $100 \mathrm{~nm}$ could be easily inserted into the branching sugar chains of mucin and thereby led to a stronger mucoadhesive property, compared to NLCs with a particle size of 200 and $300 \mathrm{~nm}$ [11]. This could be attributed to the small particle size exhibits more surface area to adhere on the corneal surfacea, In addition, surface modification of NLCs by coating with PEG could further improve ocular mucoadhesion. As Kashanian et al., noted that NLCs coating with PEG leads to an increment of nanoparticles penetration through mucus layer of the ocular surface possibly via PEG interpenetrating the mucus network aided by hydrogen bonding [40-42].

As a first step, we assessed the in vitro cytotoxicity of IND-NLCs in PCE cells following STE test protocol recommended as a potential alternative method for the assessment of ocular irritation in place of animal testing [20]. We have found the two candidates NLCs formulations (IND-NLC2 and IND-NLC2-PEG) caused much less toxicity compared to IND in solutions. Interestingly, IND nanoparticles eye drops (containing $0.5 \%$ indomethacin) prepared using zirconia beads and Bead Smash 12, the particle size of $76 \pm 59$ $\mathrm{nm}$, are much tolerated better by a human cornea epithelial cell line (HCE-T) and rat corneal epithelial cells than commercial IND eye drops [5]. This could be explained by its sustained release, which lowers the risk of locally high concentrations, decreasing the direct cells stimulation leads to minimize local irritation. Therefore, the releasing of IND from IND-NLCs to the cells provided a less toxicity compared to direct cells stimulation form IND-solutions. Moreover, the nanoparticle formulations may decrease in the drug dose via an increase in bioavailability, thus resulting in a reduction in drug toxicity. Furthermore, IND-NLCs can be easily developed avoiding the use of organic solvents, and the selection of Tween 80 as a surfactant was reported to be non-irritating to the rabbit eye up to a concentration of $10 \%$ and has been used in a number of marketed ophthalmic solution eye drop [43].

\section{CONCLUSION}

IND-NLCs were successfully prepared by a high-pressure homogenization technique to overcome the problems of IND on the topical ophthalmic formulations. Additionally, NLCs are solid at room and body temperature which can be formulated as nanodispersions in liquid dosage forms. Therefore, they can be administered as an eye drop to avoid blurred vision and comfortable due to the nano size. The developed NLCs with Tween 80 could be sterile by the autoclaving method. The particle size of $\sim 300$ and $\sim 40$ $\mathrm{nm}$ was produced depending on the type of solid lipid. Small and PEG-coated IND-NLCs enhance the mucoadhesion on the freshly porcine cornea which expected to improve ocular bioavailability. In addition, the developed NLCs showed less cytotoxicity to PCE cells compared to IND solution. Therefore, these nanoparticles will show increased effectiveness in treating ocular inflammation requiring the long-term application of eye drop with less toxicity. In conclusion, our findings suggest that NLCs with small size and PEG-coating demonstrate a promising approach for ocular drug delivery.

\section{ACKNOWLEDGEMENTS}

This work was financially supported by the Thailand Research Fund (TRF) and Naresuan University under the Royal Golden Jubilee Ph. D. grant no. PHD/0086/2556; the Naresuan University grant no. R2558B040; and Commission on High Education, Ministry of Education, Thailand under the Center of Excellence for Innovation in Chemistry (PERCH-CIC).

\section{AUTHORS CONTRIBUTIONS}

All the authors have contributed equally

\section{CONFLICT OF INTERESTS}

The authors declare that there is no conflict of interest

\section{REFERENCES}

1. Weber M, Kodjikian L, Kruse FE, Zagorski Z, Allaire CM. Efficacy and safety of indomethacin $0.1 \%$ eye drops compared with ketorolac $0.5 \%$ eye drops in the management of ocular inflammation after cataract surgery. Acta Ophthalmol 2013;91:e15-21. 
2. Vulovic N, Primprac M, Stupar M, Ford JL. Some studies into the properties of indomethacin suspensions intended for ophthalmic use. Int J Pharm 1989;55:123-8.

3. Ahuja M, Dhake AS, Sharma SK, Majumdar DK. Topical ocular delivery of NSAIDs. AAPS J 2008;10:229-41.

4. Hippalgaonkar K, Adelli GR, Hippalgaonkar K, Repka MA, Majumdar S. Indomethacin-loaded solid lipid nanoparticles for ocular delivery: development, characterization, and in vitro evaluation. J Ocul Pharmacol Ther 2013;29:216-28.

5. Nagai N, Ito $\mathrm{Y}$, Okamoto $\mathrm{N}$, Shimomura $\mathrm{Y}$. A nanoparticle formulation reduces the corneal toxicity of indomethacin eye drops and enhances its corneal permeability. Toxicology 2014;319:53-62.

6. Ammar HO, Salama HA, Ghorab M, Mahmoud AA. Nanoemulsion as a potential ophthalmic delivery system for dorzolamide hydrochloride. AAPS PharmSciTech 2009;10:808-19.

7. Balguri SP, Adelli GR, Majumdar S. Topical ophthalmic lipid nanoparticle formulations (SLN, NLC) of indomethacin for delivery to the posterior segment ocular tissues. Eur J Pharm Biopharm 2016;109:224-35.

8. Andonova V, Zagorchev P, Katsarov P, Kassarova M. Eye drops with nanoparticles as drug delivery system. Int J Pharm Pharm Sci 2015;7:431-5.

9. Battaglia L, Serpe L, Foglietta F, Muntoni E, Gallarate M, Del Pozo Rodriquez A, et al. Application of lipid nanoparticles to ocular drug delivery. Expert Opin Drug Delivery 2016;13:1743-57.

10. Sánchez-López E, Espina M, Doktorovova S, Souto EB, Garcia ML. Lipid nanoparticles (SLN, NLC): Overcoming the anatomical and physiological barriers of the eye-part II-ocular drug-loaded lipid nanoparticles. Eur J Pharm Biopharm 2017;110:58-69.

11. Li H, Chen M, Su Z, Sun M, Ping Q. Size-exclusive effect of nanostructured lipid carriers on oral drug delivery. Int J Pharm 2016;511:524-37.

12. Alvarez-Trabado J, Diebold Y, Sanchez A. Designing lipid nanoparticles for topical ocular drug delivery. Int J Pharm 2017;532:204-17.

13. Beloqui A, Solinis MA, Rodriguez-Gascon A, Almeida AJ, Preat V. Nanostructured lipid carriers: promising drug delivery systems for future clinics. Nanomedicine 2016;12:143-61.

14. Novakova L, Matysova L, Havlikova L, Solich P. Development and validation of HPLC method for determination of indomethacin and its two degradation products in the topical gel. J Pharm Biomed Anal 2005;37:899-905.

15. Niamprem P, Rujivipat S, Tiyaboonchai W. Development and characterization of lutein-loaded SNEDDS for enhanced absorption in caco-2 cells. Pharm Dev Technol 2014;19:735-42.

16. El-Badry M, Fetih G, Fathy M. Improvement of solubility and dissolution rate of indomethacin by solid dispersions in Gelucire 50/13 and PEG4000. Saudi Pharm J 2009;17:217-25.

17. Chaiyasan W, Srinivas SP, Tiyaboonchai W. Mucoadhesive chitosan-dextran sulfate nanoparticles for sustained drug delivery to the ocular surface. J Ocul Pharmacol Ther 2013;29:200-7.

18. Takahashi H, Tajima K, Hattori T, Yamakawa N, Ito N, Goto H. Novel primary epithelial cell toxicity assay using porcine corneal explants. Cornea 2015;34:567-75.

19. Chaiyasan W, Praputbut S, Kompella UB, Srinivas SP, Tiyaboonchai W. Penetration of mucoadhesive chitosandextran sulfate nanoparticles into the porcine cornea. Colloids Surf B 2017;149:288-96.

20. Wroblewska K, Kucinska M, Murias M, Lulek J. Characterization of new eye drops with choline salicylate and assessment of their irritancy by in vitro short time exposure tests. Saudi Pharm J 2015;23:407-12.

21. Sakaguchi H, Ota N, Omori T, Kuwahara H, Sozu T, Takaqi Y, et al. Validation study of the short time exposure (STE) test to assess the eye irritation potential of chemicals. Toxicol In Vitro 2011;25:796-809.

22. Takahashi Y, Hayashi T, Watanabe S, Hayashi K, Koike M, Aisawa $\mathrm{N}$, et al. Inter-laboratory study of short time exposure (STE) test for predicting eye irritation potential of chemicals and correspondence to globally harmonized system (GHS) classification. J Toxicol Sci 2009;34:611-26.

23. Araújo J, Gonzalez E, Egea MA, Garcia ML, Souto EB. Nanomedicines for ocular NSAIDs: safety on drug delivery. Nanomedicine 2009;5:394-401.

24. Stroobants A, Fabre K, Maudgal PC. Effect of non-steroidal antiinflammatory drugs (NSAID) on the rabbit corneal epithelium studied by scanning electron microscopy. Bull Soc Belge Ophtalmol 2000;276:73-81.

25. Doktorovova S, Souto EB, Silva AM. Nanotoxicology applied to solid lipid nanoparticles and nanostructured lipid carriers-a systematic review of in vitro data. Eur J Pharm Biopharm 2014;87:1-18.

26. Müller RH, Radtke M, Wissing SA. Solid lipid nanoparticles (SLN) and nanostructured lipid carriers (NLC) in cosmetic and dermatological preparations. Adv Drug Delivery Rev 2002;54 Suppl:S131-S55.

27. Huang ZR, Hua SC, Yang YL, Fang JY. Development and evaluation of lipid nanoparticles for camptothecin delivery: a comparison of solid lipid nanoparticles, nanostructured lipid carriers, and lipid emulsion. Acta Pharmacol Sin 2008;29:1094102.

28. Seyfoddin A, Shaw J, Al-Kassas R. Solid lipid nanoparticles for ocular drug delivery. Drug Delivery 2010;17:467-89.

29. Seyfoddin A, Al-Kassas R. Development of solid lipid nanoparticles and nanostructured lipid carriers for improving ocular delivery of acyclovir. Drug Dev Ind Pharm 2013;39:50819.

30. Thang LQ, Hanh ND, Duong DQ. Study on cause-effect relations and optimization of exemestane-loaded nanostructured lipid carriers. Int J Pharm Pharm Sci 2017;9:68-74.

31. Jenning V, Thünemann AF, Gohla SH. Characterisation of a novel solid lipid nanoparticle carrier system based on binary mixtures of liquid and solid lipids. Int J Pharm 2000;199:167-77.

32. Muchtar S, Abdulrazik M, Frucht-Pery J, Benitaa S. Ex-vivo permeation study of indomethacin from a submicron emulsion through albino rabbit cornea. J Controlled Release 1997;44:5564.

33. Paliwal R, Rai S, Vaidya B, Khatri K, Goyal AK, Mishra N, et al. Effect of lipid core material on characteristics of solid lipid nanoparticles designed for oral lymphatic delivery. Nanomedicine 2009;5:184-91.

34. Duarah S, Pujari K, Durai RD, Narayanan VHB. Nanotechnologybased cosmeceuticals: a review. Int J Appl Pharm 2016;8:8-12.

35. Baudouin C, Labbe A, Liang H, Pauly A, Brignole-Baudouin F. Preservatives in eyedrops: the good, the bad and the ugly. Prog Retin Eye Res 2010;29:312-34.

36. MH, Gowda DV, Vindru J, Moin A. Nanotechnology for ophthalmic preparations. Int J Curr Pharm Res 2016;8:5-11.

37. Pardeike J, Weber S, Matsko N, Zimmer A. Formation of a physical stable delivery system by simply autoclaving nanostructured lipid carriers (NLC). Int J Pharm 2012;439:22-7.

38. Ibrahim SS, Awad GAS, Geneidi A, Mortada ND. Comparative effects of different cosurfactants on sterile prednisolone acetate ocular submicron emulsions stability and release. Colloids Surf B 2009;69:225-31.

39. Gupta H, Aqil M, Khar RK, Ali A, Bhatnagar A, Mittal G. Sparfloxacin-loaded PLGA nanoparticles for sustained ocular drug delivery. Nanomedicine 2010;6:324-33.

40. Kashanian S, Rostami E. PEG-stearate coated solid lipid nanoparticles as levothyroxine carriers for oral administration. J Nanopart Res 2014;16:1-10.

41. De Campos AM, Sanchez A, Gref R, Calvo P, Alonso MJ. The effect of a PEG versus a chitosan coating on the interaction of drug colloidal carriers with the ocular mucosa. Eur J Pharm Sci 2003;20:73-81.

42. Deore S, Shahi SR, Dabir P. Nanoparticle: as targeted drug delivery system for depression. Int J Curr Pharm Res 2016;8:711.

43. Jiao J. Polyoxyethylated nonionic surfactants and their applications in topical ocular drug delivery. Adv Drug Delivery Rev 2008;60:1663-73. 